Rabaska

Revue d'ethnologie de l'Amérique française

\title{
Voyage en Acadie et autres pérégrinations nord-américaines de Geneviève Massignon : l'Amérique française vue par une " Française de France " au milieu du $\mathrm{XX}^{\mathrm{e}}$ siècle Travels in Acadia and other North American treks of Geneviève Massignon : French speaking America as seen by a French woman in the middle of the twentieth century
}

\section{Claude Verreault}

Volume 3, 2005

URI : https://id.erudit.org/iderudit/201710ar

DOI : https://doi.org/10.7202/201710ar

Aller au sommaire du numéro

Éditeur(s)

Société québécoise d'ethnologie

ISSN

1703-7433 (imprimé)

1916-7350 (numérique)

Découvrir la revue

Citer cet article

Verreault, C. (2005). Voyage en Acadie et autres pérégrinations nord-américaines de Geneviève Massignon : l’Amérique française vue par une " Française de France " au milieu du XX ${ }^{\mathrm{e}}$ siècle. Rabaska, 3, 59-69. https://doi.org/10.7202/201710ar
Résumé de l'article

Née à Paris le 21 avril 1921, Geneviève Massignon est décédée prématurément le 9 juin 1966, à l'âge de 45 ans. Fille de l'islamologue Louis Massignon, filleule de l'écrivain Paul Claudel, elle a grandi dans un milieu d'intellectuels. Après avoir obtenu une licence ès lettres classiques à la Sorbonne, elle suivra après la guerre les conférences d'Albert Dauzat à l'École des Hautes Études, alors que ce dernier s'apprêtait à lancer le chantier du nouvel atlas linguistique de la France par régions. Intéressée à la fois par la linguistique et le folklore, elle est fascinée par l'Acadie, dont elle avait entendu parler par le violoniste Arthur Leblanc, une connaissance de la famille, ainsi que par son frère Yves Massignon qui avait commencé une étude de géographie humaine sur la haute vallée du fleuve Saint-Jean, cours d'eau qui marque la frontière entre le Nouveau-Brunswick, le Québec et l'État américain du Maine. Grâce à une bourse du ministère français des Affaires étrangères, Geneviève Massignon séjournera en Acadie en 1946-1947 pour y effectuer des enquêtes linguistiques et folkloriques qui donneront lieu à deux thèses de doctorat soutenues à l'Université de Paris. Intitulée Les Parlers français d'Acadie et publiée en 1962, sa thèse principale constitue une étude remarquable à tous égards, pour laquelle Massignon a d'ailleurs obtenu la Médaille de l'Académie canadienne-française en 1963. Consacrée à la chanson populaire, sa thèse complémentaire ne sera publiée qu'en 1994. De retour en France en 1947, Geneviève Massignon se consacrera à la préparation et à réalisation de l'Atlas linguistique et ethnographique de l'Ouest. À l'occasion de son séjour en Acadie en 1946-1947, Geneviève Massignon en a profité pour traverser le Canada d'est en ouest puis pour se rendre en Louisiane, ce qui lui a permis de se faire une meilleure idée de ce que l'on appelle maintenant la francophonie nord-américaine. À partir des impressions qu'elle a rapportées de son voyage en Acadie et de ses autres pérégrinations nord-américaines, je me propose, dans cet article, de dégager la vision que cette " Française de France », comme elle s'est elle-même qualifiée, a eue de l'Amérique française de l'époque.
Ce document est protégé par la loi sur le droit d'auteur. L'utilisation des services d'Érudit (y compris la reproduction) est assujettie à sa politique d'utilisation que vous pouvez consulter en ligne.

https://apropos.erudit.org/fr/usagers/politique-dutilisation/ 


\section{Terrains}

\section{Voyage en Acadie et autres pérégrinations nord-américaines de Geneviève Massignon : l'Amérique française vue par une « Française de France » au millieu du $\mathbf{X X}^{\mathrm{e}}$ siècle*}

Claude Verreault

Université Laval

Née à Paris le 21 avril 1921, Geneviève Massignon est décédée prématurément le 9 juin 1966, à l'âge de 45 ans. Fille de l'islamologue Louis Massignon, filleule de l'écrivain Paul Claudel, elle a grandi dans un milieu d'intellectuels. Après avoir obtenu une licence ès lettres classiques à la Sorbonne, elle « apprend, seule, ce qu'il faut de finnois et de polonais pour correspondre avec ses "filleuls de guerre" polonais et finlandais en 1939-40. [...] En mai et juin 1945, elle suit les conférences du Professeur Albert Dauzat à l'École des Hautes Études ${ }^{1}$ "), alors que ce dernier s'apprêtait à lancer le chantier du nouvel atlas linguistique de la France par régions. Intéressée à la fois par la linguistique et le folklore, elle est fascinée par l'Acadie, dont elle avait entendu parler par le violoniste Arthur Leblanc, une connaissance de la famille, ainsi que par son frère Yves Massignon; ce dernier, sur les conseils du géographe Pierre Deffontaines, « avait commencé une étude de géographie humaine [...] sur la haute vallée du fleuve Saint-Jean ${ }^{2} »$, cours d'eau qui marque la frontière entre le Nouveau-Brunswick, le Québec et l'État américain du Maine.

Grâce à une bourse du ministère français des Affaires étrangères, Geneviève Massignon séjournera en Acadie en 1946-1947 pour y effectuer des enquêtes linguistiques et folkloriques qui donneront lieu à deux thèses de doctorat soutenues à l'Université de Paris. Intitulée Les Parlers français

* Version remaniée d'une communication présentée à Paris le 2 février 1999, à l'occasion d'une journée scientifique consacrée à l'œuvre de Geneviève Massignon ; organisée par Marie-Rose Simoni et Liselotte Pasques, rattachées au Centre national de la recherche scientifique et à l'Université de Paris III, avec la participation de Jacques Chaurand, professeur émérite à l'Université de Paris XIII, cette journée s'est tenue dans le cadre du séminaire Histoire de la langue et structure de l'orthographe, dialectologie.

1. Daniel Massignon, « Place de l'Acadie dans l'œuvre de Geneviève Massignon », dans Geneviève Massignon, Trésors de la chanson populaire française. Autour de 50 chansons recueillies en Acadie, vol. 1 (Textes), Paris, Bibliothèque nationale de France, 1994, p. vii et ix.

2. Ibid., p. vii. 
d'Acadie et publiée en $1962^{3}$, sa thèse principale constitue une étude remarquable à tous égards ${ }^{4}$, pour laquelle Massignon a d'ailleurs obtenu la Médaille de l'Académie canadienne-française en $1963^{5}$. Consacrée à la chanson populaire, sa thèse complémentaire ne sera publiée qu'en $1994^{6}$.

De retour en France en 1947, Geneviève Massignon se consacrera à la préparation et à la réalisation de l'Atlas linguistique et ethnographique de l'Ouest ${ }^{7}$ :

Enquêteuse bénévole depuis 1947, puis comme ingénieur au C.N.R.S. [Centre national de la recherche scientifique], de 1957 à sa mort, elle travaille[,] à la demande d'Albert Dauzat[,] avec le Professeur Jacques Pignon, spécialiste du Poitou, à la préparation du questionnaire de l'un des 19 Atlas régionaux du Nouvel Atlas Linguistique de la France, l'Atlas Linguistique de l'Ouest. La « région de l'Ouest » couverte par cet Atlas est délimitée par la Loire, les confins Occidentaux du Massif Central, la Dordogne-Garonne et l'Atlantique. Elle contient, en particulier, le Sud de l'Indre-et-Loire, le Poitou, l'Aunis et la Saintonge, dont on sait que sont originaires des familles Acadiennes [sic]. Très rapidement, Jacques Pignon, trop occupé par ailleurs, lui en laisse la pleine responsabilité.

Elle met alors au point un questionnaire linguistique de 3500 mots, basé sur les enquêtes préliminaires commencées dans cette région dès 1945, avant son premier voyage en Acadie. De 1958 à sa mort, elle a pu visiter 124 des 135 points d'enquête prévus. ${ }^{8}$

À l'occasion de son séjour en Acadie en 1946-1947, Geneviève Massignon en a profité pour traverser le Canada d'est en ouest puis pour se rendre en Louisiane, ce qui lui a permis de se faire une meilleure idée de ce que l'on appelle maintenant la francophonie nord-américaine. À partir des impressions qu'elle a rapportées de son voyage en Acadie et de ses autres pérégrinations nord-américaines, je me propose, dans cet article, de dégager

3. Geneviève Massignon, Les Parlers français d'Acadie. Enquête linguistique, Paris, Librairie C. Klincksieck, 1962, 975 p. en 2 vol. Épuisé depuis plusieurs années, cet ouvrage sera bientôt réédité aux Presses de l'Université Laval, dans la collection « Langue française en Amérique du Nord ».

4. Claude Verreault, " Variation géographique du français dans l'Est du Canada. Présentation des principales enquêtes réalisées et aperçu des recherches actuelles ", dans Michel Contini (dir.), $L a$ Géolinguistique en Amérique latine, Grenoble, Centre de dialectologie de l'Université StendhalGrenoble III, 2002, p. 247-248.

5. Jean Royer, Chronique d'une Académie, 1944-1994. De l'Académie canadienne-française à l'Académie des lettres du Québec, Montréal, L'Hexagone, 1995, p. 75 et 149.

6. Geneviève Massignon, Trésors de la chanson populaire française. Autour de 50 chansons recueillies en Acadie, vol. 1 (Textes), Paris, Bibliothèque nationale de France, 1994, lvi-373 p. ; vol. 2 (Mélodies), $161 \mathrm{p}$.

7. Geneviève Massignon et Brigitte Horiot, Atlas linguistique et ethnographique de l'Ouest (Poitou, Aunis, Saintonge, Angoumois), vol. 1, Paris, Éditions du Centre national de la recherche scientifique (" Atlas linguistiques de la France par régions »), 1971 ; vol. 2, 1974 ; vol. 3, 1983.

8. Daniel Massignon, op. cit., p. xix. 
la vision que cette "Française de France ", comme elle s'est elle-même qualifiée', a eue de l'Amérique française de l'époque.

\section{La communauté acadienne}

De toutes les communautés françaises vivant et se développant en Amérique du Nord, la communauté acadienne est à n'en pas douter celle que Geneviève Massignon connaissait le mieux, notamment en raison des enquêtes qu'elle y avait effectuées et de l'excellente connaissance qu'elle avait de son histoire. Dans un article paru en $1947^{10}$, elle se révèle en effet non seulement une dialectologue chevronnée mais aussi une excellente historienne ; à ses yeux, on ne peut bien comprendre la situation linguistique des Acadiens sans avoir au préalable une idée des principaux événements historiques qui ont contribué à façonner ce peuple. Ainsi, après avoir établi que le peuple acadien est issu d'un tout petit groupe de colons français venus s'établir à Port-Royal (aujourd'hui Annapolis, en Nouvelle-Écosse) au XVII ${ }^{\mathrm{e}}$ siècle, elle rappelle comment la déportation dont il fut l'objet au XVIII' siècle est responsable de sa dissémination subséquente le long des côtes canadiennes de l'Atlantique, en particulier de celles du Nouveau-Brunswick, ce qui n'a pas été sans conséquence sur le plan linguistique :

[...] l'examen de la carte géographique montrant la distribution du peuplement acadien fait voir qu'il est réparti en une série de « noyaux » séparés les uns des autres par des éléments anglo-saxons plus nombreux ou par de vastes zones non peuplées. Cette particularité explique à la fois certains régionalismes au sein de l'acadien lui-même, dont les rameaux ont évolué séparément, et l'influence de l'anglicisme, surtout dans les "îlots" les plus dispersés. [...] il y a des régionalismes, « à petite échelle », qui permettent [...] à un Acadien de la Baie Sainte-Marie (sud de la Nouvelle-Écosse) de dire à un Acadien du Cap Breton : «à ton parler, je vois que tu es de Chéticamp !... »"

Bien avant l'avènement de la dialectométrie et de ses savants et coûteux calculs, Massignon avait donc mis en évidence à la fois l'uniformité et la diversité des parlers acadiens. Elle avait aussi bien vu que le parler des Acadiens établis dans la vallée du Madawaska (nord-ouest du NouveauBrunswick) tenait davantage du parler des Canadiens (ou des Québécois, comme on dirait aujourd'hui) que du parler typiquement acadien ${ }^{12}$. L'importance que Massignon accordait à l'histoire se manifeste également

9. Geneviève Massignon, «L'Ouest en Congrès ", Vie française, Québec, $\mathrm{n}^{\circ} 1$ (octobre 1946), p. 36 .

10. Geneviève Massignon, « Les Parlers français d'Acadie ", The French Review, Baltimore, vol. 21, $\mathrm{n}^{\circ} 1$ (octobre 1947), p. 45-53.

11. Ibid., p. 46.

12. Ibid., p. 45 . 
dans deux autres articles respectivement parus en $1963^{13}$ et en $1964^{14}$, dans lesquels elle démontre que bon nombre des pionniers venus s'établir en Acadie au XVII ${ }^{e}$ siècle étaient originaires de la région de Loudun, ce qui explique une bonne partie des différences qui existent entre les parlers acadiens et québécois.

Aussitôt ses enquêtes sur le terrain terminées, c'est-à-dire dès 1947, Massignon était en mesure de dégager les principaux traits linguistiques caractéristiques des parlers acadiens ${ }^{15}$.

Pour ce qui est de la prononciation, elle mentionne notamment les traits suivants : finale infinitive -oir prononcée [we:r] (par exemple, avoir et savoir prononcés [avwe:r] et [savwe:r]) ; finale substantive -oir prononcée [we] (par exemple, mouchoir et rasoir prononcés [mufwe] et [razwe]); hésitation entre [o] et [u] dans des mots comme homme, prononcé [om] ou [um], comme c'était le cas en France encore au XVII ${ }^{\mathrm{e}}$ siècle ; palatalisation de [k] en [t $\left.\mathrm{f}\right]$ et de $[\mathrm{d}]$ en [ $\mathrm{d} 3]$ devant certaines voyelles (par exemple, curé prononcé [ $\left.\mathrm{t} \int \mathrm{yre}\right]$, et gueule prononcé [dzœl]), trait qui est attesté aussi en Saintonge et dans le Poitou.

Parmi les traits morphologiques qu'elle relève, signalons les suivants : infinitif en -zir plutôt qu'en -ir (par exemple, vieillezir et blêmezir pour vieillir et blêmir); emploi de je à la place de nous à la première personne du pluriel (par exemple, $j$ 'allions et $j$ 'venons pour nous allions et nous venons) ; maintien du passé simple, régularisé en $-i$ (par exemple, il tombit et ils jetirent) ; emploi de l'auxiliaire avoir à la place de l'auxiliaire être, trait attesté également dans les parlers populaires du Québec (par exemple, il a venu et $i l$ a parti pour il est venu et il est parti); présence de certains participes passés irréguliers (par exemple, vi pour vécu, et mouri ou mouru pour mort). Dans ce dernier cas, il est intéressant de noter que Massignon a bien vu le rapport de concurrence et d'opposition qui existe entre mouri ou mouru d'une part, et mort d'autre part : «Dans il est mort, acadien [il $\varepsilon$ mor], mort fait fonction d'adjectif $^{16} \gg$.

Pour la syntaxe, qu'elle qualifie de « populaire ${ }^{17} »$, elle signale notamment les deux traits suivants : emploi de la particule interrogative ou exclamative $t i$ (par exemple, [f $\varepsilon$ : ti Jo] pour comme il fait chaud!), trait bien connu également au Québec ; emploi de la particule négative point à la place de pas (par exemple, il l'a point connu).

13. Geneviève Massignon, « La Seigneurie de Charles de Menou d'Aulnay, gouverneur de l'Acadie, 1635-1650 ", Revue d'histoire de l'Amérique française, Montréal, vol. 16, n 4 (mars 1963), p. 469-501.

14. Geneviève Massignon, "Les Trahan d'Acadie ", La Société historique acadienne, Moncton, $5^{\mathrm{e}}$ cahier, 1964, p. 10-23.

15. Geneviève Massignon, « Les Parlers français d'Acadie ", op.cit.

16. Ibid., p. 49.

17. Ibid., p. 50. 
Quant au lexique, Massignon précise que le vocabulaire acadien « est celui du langage populaire en France ${ }^{18} "$, mais que ce vocabulaire populaire a été « rétréci par une séparation trois fois séculaire de sorte qu'il y manque beaucoup de termes de la vie moderne, et s'y trouve[nt] bien des anglicismes qui ne les remplacent $\operatorname{pas}^{19} »$. Du point de vue de ses origines, Massignon signale que le vocabulaire acadien est fait d'archaïsmes (par exemple, septante « soixante-dix » et mitan « milieu »), de dialectalismes hérités principalement des parlers de 1'Ouest et du Centre-Ouest français (par exemple, remeuil " pis de la vache », subler " siffler » et baille " cuve "), sans compter les anglicismes dont elle ne donne cependant aucun exemple (on sait qu'elle a exclu de son étude parue en 1962 tous les mots d'origine anglaise). Massignon souligne par ailleurs l'influence que le vocabulaire maritime a eue sur le vocabulaire acadien, influence sur laquelle elle reviendra à plusieurs reprises ${ }^{20}$. Enfin, elle se montre encore une fois sensible au phénomène de concurrence, ici lexicale :

[...] bailler et donner conservent leurs domaines respectifs, le premier : donner au sens d'apporter : baille-moi ça, le second, donner au sens de faire cadeau : me donnes-tu ça ? dans la région de la Baie Sainte-Marie, Nouvelle-Écosse. ${ }^{21}$

Pour illustrer le parler acadien, la dialectologue n'hésite pas à céder le pas à la folkloriste qui a cru bon reproduire un conte qu'elle avait recueilli à la Baie-Sainte-Marie en Nouvelle-Écosse. Mais aussitôt chassée, la dialectologue revient au galop pour insister sur le fait que la langue de ce conte est " accessible à tous ceux qui savent le français ${ }^{22}$ ", ce qui revient à dire que Massignon considérait bel et bien le parler acadien comme une variété de français et non comme un dialecte distinct, point de vue très avant-gardiste pour l'époque, surtout de la part d'une dialectologue formée à l'école française.

En 1948, Geneviève Massignon publiait un autre article ${ }^{23}$ qui aide à mieux situer le vocabulaire qu'elle a recueilli dans ses enquêtes. Dans ce texte en effet, on constate que la dialectologue se confondait souvent avec l'ethnologue et la folkloriste :

18. Ibid., p. 51.

19. Ibid., p. 51.

20. Geneviève Massignon, « Voyage en Acadie », Le Trait d'union, Québec, vol. 2, n 4 (avril 1947), p. 4 ; «Impressions de voyage en Louisiane », Vie française, Québec, $n^{\circ} 9$ (juin-juillet 1947), p. 577 ; «Les Minorités françaises au Canada », Le Français dans le monde, Paris, ${ }^{\circ} 23$ (mars 1964 : Le Canada français ; numéro spécial), p. 19.

21. Geneviève Massignon, «Les Parlers français d'Acadie », op. cit., p. 51.

22. Ibid., p. 53.

23. Geneviève Massignon, « Le Folklore de l'Acadie », Le Trait d'union, Québec, vol. 3, n 5 (juin 1948), p. 1-2. 
Avec la langue du XVII siècle, dont l'Acadien a si bien conservé la forme et l'accent, les premiers colons ont apporté jadis leurs croyances, leurs coutumes, leurs veillées, leurs contes, leurs chansons et jusqu'aux moindres jeux dont les enfants de langue française font partout leurs délices : tel le jeu de colin-maillard dont j'ai recueilli neuf appellations différentes lors de mes enquêtes folkloriques en Acadie. ${ }^{24}$

À lire les nombreux exemples qu'elle mentionne (lessi n. f. « lessive" ; foulerie « réunion au cours de laquelle on s'adonnait au foulage des étoffes nouvellement tissées »; javelier " sorte de faux munie d'un râteau "; veriou " premier lait donné par une vache qui vient de vêler »), on peut penser qu'une bonne partie du vocabulaire qu'elle a relevé dans ses enquêtes référait à des traditions sinon révolues du moins en voie de l'être, et était par conséquent sans doute déjà désuet.

L'affection, pour ne pas dire l'amour, que Massignon portait à la communauté acadienne a donné lieu à un texte touchant paru en $1947^{25}$. Rédigé après son retour en France, ce texte est l'occasion pour elle de se remémorer les étapes les plus importantes de son séjour en Acadie et de rendre hommage aux femmes et aux hommes qu'elle avait côtoyés et pour qui elle se plaisait à imaginer une « Acadie future [...] plus que jamais catholique et françai$\mathrm{se}^{26} \gg$. Cette vision très idéalisée tranche avec le constat qu'elle fera quelque vingt ans plus tard. En effet, dans un texte paru en 1964 portant sur les minorités françaises du Canada ${ }^{27}$, Massignon fera preuve de beaucoup plus de réalisme. Ainsi, malgré les statistiques qui montrent un accroissement constant de la population d'origine française en Acadie, elle restera lucide :

$[\ldots]$ ces statistiques sont à corriger par celles des langues « maternelle » ou " officielle ", qui permettent de constater la perte de l'usage du français chez un pourcentage d'Acadiens, variable, selon leur isolement ou leur dispersion au sein d'une masse anglophone (en Nouvelle-Écosse et dans l'île du PrinceÉdouard surtout). ${ }^{28}$

\section{La communauté québécoise}

Geneviève Massignon n'a pas consacré de texte particulier à la communauté d'origine française vivant dans « la province de Québec », comme on désignait alors couramment le Québec, mais on peut glaner ici et là quelques commen-

24. Ibid., p. 1. Pour jouer à colin-maillard, elle a en fait relevé huit expressions différentes : jouer aux beaux-yeux, jouer aux yeux bouchés, jouer à boucher les yeux, jouer à chat mouillé, jouer au petit chien, jouer à colin-maillard, jouer au cordonnier et jouer à la morue (cf. Les Parlers français d'Acadie. Enquête linguistique, Paris, Librairie C. Klincksieck, 1962, question $n^{\circ} 1848$ ).

25. Geneviève Massignon, « Voyage en Acadie », op. cit., p. 4.

26. Ibid., p. 4.

27. Geneviève Massignon, «Les Minorités françaises au Canada », op. cit., p. 17-21.

28. Ibid., p. 19. 
taires qui laissent entrevoir la perception qu'elle pouvait en avoir. À ses yeux, le Québec constituait un cas à part du seul fait que les francophones y étaient majoritaires. Elle déplore d'ailleurs le fait que les jeunes francophones nés ailleurs au Canada ne connaissent pas, et ne connaîtront sans doute jamais, l'ambiance toute française qui caractérise cette province ${ }^{29}$.

Pour expliquer les nombreuses différences existant entre le parler du Québec et celui de l'Acadie, Massignon recourt de nouveau à l'histoire :

Le recrutement des colons ne fut pas le même pour l'Acadie et pour le Canada ; d'importants éléments venus de Normandie, du Maine, du Perche, d'île-deFrance et du Val de Loire précédèrent l'élément poitevin au Canada, tandis qu'en Acadie, les éléments angevins, poitevins, aunisiens et saintongeais sont prédominants $[\ldots] .^{30}$

Pour ce qui est des traits caractéristiques du parler québécois, elle se limite à l'observation suivante, qui est du reste plutôt un jugement de nature épilinguistique :

[...] leur langue [celle des familles acadiennes], dont l'accent, sans cesser d'avoir la saveur du terroir, est moins "provincial » que celui de bien des villages québecois $\left[\right.$ sic.$^{31}$

\section{La communauté ontarienne}

Dans un article paru en 1964, Massignon rappelle d'abord que l'Ontario français est "une expansion du Québec, aux nombreux jeunes foyers surpeuplés ${ }^{32} »$. Cette remarque n'est pas fausse, mais elle mériterait d'être nuancée ; en effet, les recherches récentes ont établi que de nombreux Acadiens sont également venus peupler l'Ontario, tant au XIX ${ }^{\mathrm{e}}$ qu'au $\mathrm{XX}^{\mathrm{e}}$ siècle $^{33}$.

Si les Franco-Ontariens disposent d'écoles et même « d'une université où la langue française est à l'honneur ${ }^{34} »$, Geneviève Massignon estime que cela « ne peut pallier les effets néfastes d'une législation officielle ontarienne,

29. Geneviève Massignon, «L'Ouest en Congrès ", op. cit., p. 37.

30. Geneviève Massignon, "Les Minorités françaises au Canada ", op. cit., p. 18.

31. Geneviève Massignon, "Voyage en Acadie ", op. cit., p. 4.

32. Geneviève Massignon, "Les Minorités françaises au Canada ", op. cit., p. 19.

33. Cf. notamment Raymond Mougeon et Édouard Beniak, Linguistic Consequences of Language Contact and Restriction. The Case of French in Ontario, Canada, Oxford, Clarendon Press ("Oxford Studies in Language Contact »), 1991, 247 p. ; Serge Wagner, avec la collab. de Pierre Grenier, Analphabétisme de minorité et alphabétisation d'affirmation nationale. Á propos de l'Ontario français, vol. 1 (Synthèse théorique et historique), [Toronto], Ministère de l'Éducation, Unité de l'alphabétisation communautaire et en milieu de travail (" Alpha communautaire chez les Franco-Ontariens, 9 »), 1990, xii-506 p.

34. Geneviève Massignon, "Les Minorités françaises au Canada ", op. cit., p. 20. 
pour qui le bilinguisme n'est pas une préoccupation majeure ${ }^{35}$ ". À ce propos, elle va même jusqu'à établir certaines comparaisons entre les situations ontarienne et québécoise :

On est surpris de constater combien peu de citoyens ontariens d'origine britannique connaissent le français, alors que tant de citoyens d'origine française connaissent l'anglais au Québec, où les établissements d'enseignement britanniques peuvent pleinement s'épanouir. ${ }^{36}$

\section{Les communautés des provinces de l'Ouest canadien}

Geneviève Massignon a traité de ces communautés dans deux articles différents, le premier étant paru en $1946^{37}$ et le deuxième en $1964^{38}$.

Dans le premier, Massignon relate le « long périple [qu'elle a] accompli à travers l'Ouest canadien ${ }^{39}$ " à l'été 1946 et qui lui permit de se faire une meilleure idée des conditions de vie et d'épanouissement des communautés francophones établies dans cette partie du pays.

Comme elle le souligne très justement, ces communautés françaises sont issues de groupes d'origines diverses ; à côté des Canadiens français venus de l'Est du pays, on trouve aussi des « Français de France ${ }^{40}$ » et des Belges.

Bien qu'elle reconnaisse que ces communautés soient largement minoritaires et très dispersées sur un vaste territoire, Massignon est d'avis que le fort taux de natalité qui les caractérise devrait garantir leur maintien :

La vitalité, on pourrait dire la fécondité de la race française au Canada compense, ici comme ailleurs, les pertes subies, lesquelles sont dues à l'école et à 1'atmosphère anglicisatrices. ${ }^{41}$

À propos de l'éducation en français, on est frappé par l'ambiguïté des propos de Massignon. D'une part, elle semble relativement optimiste face à la situation qui a cours au Manitoba :

L'Église avec ses couvents, 128 écoles, est comme le centre de la vie française là-bas. Il a fallu batailler pour obtenir que la langue française ait sa place à l'école, là où il y a des petits Canadiens-Français [sic]. Les Associations d'Éducation sont anciennes dans l'Ouest : trente, quarante ans. Elles sont aguerries, rompues à la lutte, habituées à la victoire... ${ }^{42}$
35. Ibid., p. 20.
36. Ibid., p. 20.
37. Geneviève Massignon, «L'Ouest en Congrès ", op. cit., p. 36-43.
38. Geneviève Massignon, «Les Minorités françaises au Canada », op. cit., p. 17-21.
39. Geneviève Massignon, "L'Ouest en Congrès ", op. cit., p. 36.
40. Ibid., p. 40.
41. Ibid., p. 41.
42. Ibid., p. 37. 
De l'autre, elle paraît s'inquiéter du peu de place accordée au français dans les écoles publiques auxquelles ont accès les jeunes Canadiens français de l'Ouest :

Un tableau [...] nous présente [...] la situation scolaire en Alberta : c'est à peu près la même qu'en Saskatchewan et Manitoba. Les Canadiens-Français [sic] y ont des écoles séparées, et dans les écoles publiques (là où ils sont en majorité) ils ont le régime scolaire suivant: les deux premières années tout en français. Ensuite, l'enseignement se fait tout en anglais, sauf une heure de français par jour. Il s'y ajoute une demi-heure de catéchisme par jour, en français. ${ }^{43}$

Par ailleurs, elle se réjouit de constater l'existence de certains hebdomadaires canadiens-français et elle estime que la radio française, qui faisait alors son apparition dans l'Ouest canadien, est appelée à jouer un rôle déterminant dans le maintien et l'expansion de la culture française.

Entrepris « en compagnie de nombreux délégués du Québec et de 1'Ontario ${ }^{44}$ » venus dans l'Ouest pour assister aux divers congrès provinciaux du Comité de la survivance française, ce voyage s'est somme toute déroulé dans une atmosphère de festivités et de grand débordement d'enthousiasme à l'égard de la "race française ${ }^{45}$ " essaimée dans cette partie du Canada. C'est sans doute ce qui explique la vision quelque peu idyllique que Massignon semble avoir eue des conditions de vie et de développement des communautés francophones qui y vivent, voire de leurs effectifs réels :

La population des Canadiens-Français [sic] a beaucoup augmenté en Colombie[-Britannique], on en trouve un peu partout, et les chiffres du recensement de 1941 sont très inférieurs à la réalité présente. ${ }^{46}$

Dans le deuxième article, Massignon reprend à peu près les mêmes idées, mais elle se montre beaucoup plus réaliste ou pessimiste, selon le point de vue que l'on veut bien adopter.

\section{La communauté louisianaise}

Dans un texte paru en 1947, Massignon livre ses impressions d'un voyage qu'elle a effectué en Louisiane dans le courant de l'hiver 1946-194747. Au-delà du charme, du pittoresque et de la naïveté de certaines de ses descriptions, elle rappelle tout d'abord que la présence française en Louisiane remonte à trois types distincts de peuplement :
43. Ibid., p. 41.
44. Ibid., p. 36.
45. Ibid., p. 36.
46. Ibid., p. 43.
47. Geneviève Massignon, «Impressions de voyage en Louisiane », op. cit., p. 570-578. 
Là-bas, ont pour langue maternelle le français : les descendants appelés Créoles des Français installés en Louisiane sous le régime français (XVIII ${ }^{e}$ siècle) auxquels se sont ajoutés les Acadiens, dont beaucoup, exilés en 1755, trouvèrent un refuge définitif en Louisiane; et enfin les noirs [sic], implantés dans les mêmes régions, descendants des esclaves amenés d'Afrique pour travailler dans les plantations. ${ }^{48}$

Par la suite, elle esquisse le portrait sociolinguistique de cet État américain. Ainsi, elle nous apprend que la capitale (la Nouvelle-Orléans) est « une grande ville de 500,000 habitants, [...] mais [où] le nombre des francophones se chiffre seulement par quelques milliers ${ }^{49} »$, que Lafayette est par contre une « jolie petite ville de 25,000 habitants, où le français et l'anglais sont conjointement employés partout ${ }^{50}$ » et, surtout, qu'il existe en réalité deux Louisiane : celle du Nord, anglaise, et celle du Sud, « en majorité d'origine française ${ }^{51}$ », dont elle a voulu " sonder les limites " nord" "52 », comme quoi les préoccupations dialectologiques étaient toujours présentes chez elle.

Si Massignon reconnaît certains traits linguistiques acadiens dans les diverses localités qu'elle a visitées, c'est le petit village d'Abbeville qui constitue selon elle « le cœur même de l'Acadie louisianaise ${ }^{53}$ :

Le langage [y] est bien resté acadien par les nombreuses expressions maritimes dont ces ruraux se servent "à tout bout de champ " sans bien se rendre compte de leur acception originelle : gréer, pour vêtir, une embarcation [,] pour une voiture, naviguer, pour se promener, etc. ${ }^{54}$

Lors de ce voyage, Geneviève Massignon a aussi eu l'occasion de rencontrer Hosea Phillips, auteur d'une thèse de doctorat portant sur le parler de la paroisse ${ }^{55}$ d'Évangéline (soutenue à Paris, cette thèse a été publiée en $\left.1936^{56}\right)$ et elle a été mise au courant des nombreuses enquêtes dialectologiques

48. Ibid., p. 573.

49. Loc. cit., note 1.

50. Loc. cit.

51. Loc. cit.

52. Ibid., p. 574. Cf. en outre p. 574-575 : « De Mansura, je gagne Alexandria et Natchitoches [...]. Natchitoches, un des premiers postes fondés par les Français au XVIII' siècle, est actuellement anglophone : ici commence la Louisiane du nord, qui s'annonce géographiquement par des collines. »

53. Ibid., p. 577.

54. Loc. cit.

55. En Louisiane, le mot paroisse désigne une entité administrative qui peut englober plusieurs villages et petites villes ( $c$. Dictionnaire universel francophone, AUPELF-UREF - Hachette Edicef, 1997).

56. Cf. Hosea Phillips, Étude du parler de la paroisse Évangéline (Louisiane), Paris, Librairie E. Droz (« Société de publications romanes et françaises, 17 »), 1936, iv-133 p. 
effectuées dans diverses autres paroisses dans le cadre de mémoires de maîtrise réalisés à la Louisiana State University ${ }^{57}$.

\section{En guise de conclusion}

Geneviève Massignon a porté sur l'Amérique française du milieu du XX siècle le regard de la dialectologue, de l'historienne, de l'ethnologue et de la folkloriste qu'elle était. Selon ces diverses personnalités, elle en a eu la vision tantôt idéalisée d'un doux passé enfin retrouvé, tantôt plus réaliste d'un présent troublant et d'un avenir incertain.

57. Cf. notamment Anna Theresa Daigle, Folklore and Etymological Glossary of the Variants from Standard French in Jefferson Davis Parish, mémoire de maîtrise, Louisiana State University, 1934, [178] p. ; Bertrand François De Blanc, A Glossary of Variants from Standard French Found in St. Martin Parish, mémoire de maitrise, Louisiana State University, 1935, ix-45 p. ; Alice Dugas, A Glossary of the Variants from Standard-French Used in the Parish of Saint James, mémoire de maitrise, Baton Rouge, Louisiana State University, 1935, v-59 p. ; Samuel L. Jeansonne, A Glossary of Words that Vary from Standard-French in Avoyelles Parish, mémoire de maittrise, Louisiana State University, 1938, viii-128 p. ; Ervin Louis Granier, A Glossary of the French Spoken in St. John Parish, mémoire de maîtrise, Louisiana State University, 1939, x-155 p. 